\title{
Structure and dynamics of the protoplankton community in an environmentally protected urban stream
}

\author{
M. A. M. Lipperta (D), F. M. Lansac-Tôha ${ }^{b}$ (D), B. R. Meira ${ }^{b}$ (D), L. F. M. Velho ${ }^{b, c}$ (D) and \\ F. A. Lansac-Toha ${ }^{b *}$ \\ aPrograma de Pós-graduação em Biologia Comparada, Universidade Estadual de Maringá - UEM, \\ Av. Colombo, 5790, CEP 87020-900, Maringá, PR, Brasil \\ búcleo de Pesquisas em Limnologia, Ictiologia e Aquicultura - NUPELIA, Programa de Pós-graduação em Ecologia de \\ Ambientes Aquáticos Continentais, Universidade Estadual de Maringá - UEM, \\ Av. Colombo, 5790, CEP 87020-900, Maringá, PR, Brasil \\ 'Instituto Cesumar de Ciência, Tecnologia e Inovação - ICETI, Centro Universitário Cesumar - Unicesumar, \\ Av. Guedner, 1610, CEP 87050-900, Maringá, PR, Brasil \\ *e-mail: fabio@nupelia.uem.br
}

Received: April 12, 2019 - Accepted: July 31, 2019 - Distributed: November 30, 2020

(With 10 figures)

\begin{abstract}
We aimed to investigate spatial and temporal scales, abundance, and factors that structure the communities of protozoans in a tropical urban stream. Methods: Samples of water for analysis of biological communities (testate amoebae, ciliates and bacteria) and limnological variables were taken in the Mandacaru stream located in the Conservation Unit of Parque do Cinquentenário, in the city of Maringá, Paraná State, Brazil, in two hydrological periods (dry and rainy). We calculated the Water Quality Index (WQI) composed by nine parameters of water quality (dissolved oxygen, thermotolerant coliforms, $\mathrm{pH}$, BOD, water temperature, total nitrogen, total phosphorous, turbidity, and total solids), Trophic State Index (TSI) for phosphorous and we used a Redundancy Analysis (RDA) to verify the influence of environmental variables in the protozoan community. Results: The WQI showed that water quality was considered good in some points and considered bad in other points. The TSI for phosphorus classified the stream as mesotrophic in the majority of sampled sites points (mean between 53.09 and 58.35). We identified 19 taxa of testate amoebae, belonging to six families, being Difflugiidae, Centropyxidae, and Arcellidae those with more species and 71 infrageneric taxa of ciliates, distributed in 12 orders being Peniculida the most representative order, followed by Euplotida. According to RDA analysis, samples of the dry period were characterized by higher values of bacteria density and concentrations of chlorophyll-a, total phosphorous, and total nitrogen. Ciliates and testate amoebae presented higher abundance values in some of the months characterized by higher precipitation and in conditions of higher system productivity. Conclusion: we may conclude that the Mandacaru stream, although strongly influenced by anthropic action, still presents an acceptable water quality. Lastly, we emphasize that protists abundance was strongly influenced by system productivity. This was evidenced by elevated protozoan densities where there was higher primary and bacterial productivity. Thus, these organisms must be considered in studies that aim at the identification of organisms that may indicate anthropic impacts and environmental quality.
\end{abstract}

Keywords: protist, plankton, water quality, testate amoebae, ciliates.

\section{Estrutura e dinâmica da comunidade de protoplâncton em um córrego urbano ambientalmente protegido}

\section{Resumo}

O objetivo foi investigar, em escalas espacial e temporal, a abundância das comunidades de protozoários bem como os fatores intervenientes na estruturação das mesmas em um córrego urbano. Métodos: Amostras de água para análise de comunidades biológicas (amebas testáceas, ciliados e bactérias) e para análise de variáveis limnológicas foram tomadas no córrego Mandacaru localizado na Unidade de Conservação do Parque do Cinquentenário, na cidade de Maringá, Paraná State, Brasil, em dois períodos hidrológicos (seco e chuvoso). Calculamos o Índice de Qualidade de Água (IQA), o Índice de Estado Trófico (ETI) para fósforo e utilizamos uma Análise de Redundância (RDA) para verificar a influência de variáveis ambientais na comunidade de protozoários. Resultados: O IQA mostrou que a qualidade da água foi considerada boa em alguns pontos e considerada ruim em outros pontos, e o ETI para o fósforo classificou o córrego como mesotrófico na maioria dos locais amostrados (média entre 53,09 e 58,35). Foram identificados 
19 táxons de amebas testáceas, pertencentes a seis famílias, sendo Difflugiidae, Centropyxidae e Arcellidae as mais especiosas, e 71 táxons de ciliados, distribuídos em 12 ordens, sendo Peniculida a ordem mais representativa, seguida por Euplotida. De acordo com a análise RDA, amostras do período seco foram caracterizadas por maiores valores de densidade bacteriana e concentrações de clorofila-a, fósforo total e nitrogênio total. As amebas testáceas e os ciliados apresentaram valores de abundância maiores em alguns dos meses caracterizados por maior precipitação e em condições de maior produtividade do sistema. Podemos concluir que o córrego Mandacaru, embora fortemente influenciado pela ação antrópica, ainda apresenta uma qualidade de água aceitável. Por fim, enfatizamos que a abundância de protistas foi fortemente influenciada pela produtividade do sistema. Isto foi evidenciado pelas elevadas densidades de protozoários onde houve maior produtividade primária e bacteriana. Assim, esses protistas devem ser considerados em estudos que visem à identificação de organismos que possam indicar impactos antrópicos e qualidade ambiental.

Palavras-chave: protozoários, plâncton, qualidade da água, amebas testáceas, ciliados.

\section{Introduction}

City growth in the last decades has been responsible for increased pressure of anthropic activities on natural resources (Lopes et al., 2015; Martínez-Fernández et al., 2019). Changes in habitat structure and in processes that control patterns of composition, species diversity and abundance, including specific interactions, microclimate, and resource availability, are widely reported in scientific literature as recurrent phenomena in urban landscapes (Shochat et al., 2006).

Water quality in the planet has been intensely degraded (Rebouças et al., 2002; Andrietti et al., 2016), generating loss of aquatic biodiversity due to physical and chemical restructuring of the environment and alteration of the natural dynamics of biological communities. Efforts to evaluate and manage urban aquatic resources exist in scientific, political, and social spheres (Alberti, 2009). Even if incipient, these actions seek, through the development of research that contemplates quantitative and qualitative aspects of the resources, to evaluate the integrity of these ecosystems and subsidize management actions. Rapid urban growth together with previous anthropogenic impacts promote environmental changes in small time lapses, reducing the ability of ecosystems to keep the balance driven by the action of environmental factors combined in different spatial scales (Moerke and Lamberti, 2006).

Recent studies describe the effects of urbanization on urban streams, including hydrographic changes, increase in nutrient and contaminant concentrations, and alterations in the morphology and stability of the canal (Paul and Meyer, 2001; Cunico et al., 2012; Harfuch et al., 2019). To better understand the water quality of a river, physical, chemical, and biological characteristics must be considered (Lobo et al., 2004).

Physical variables are mainly estimates of temperature, watercolor and turbidity. Chemical quality is measured by the identification, through specific methodologies, of water components as concentrations of dissolved oxygen, biochemical and chemical oxygen demands, $\mathrm{pH}$, and conductivity.

Biological communities may be good indicators of the influence of human expansion on the environment, and are important for the evaluation of water quality (Munn et al., 2002). Protoplankton is an aquatic community that represents initial levels of the trophic nets acting in matter and energy transfer, these are fundamental organisms for the dynamics of aquatic ecosystems (Fenchel, 1987; Rossi et al., 2016).

Particularly, the small size, high metabolic rates, and short life cycle allow the protists to respond rapidly to changes in environmental characteristics ( $\mathrm{Xu}$ et al., 2002; Segovia et al., 2016). These organisms are good indicators of stream health (Dias et al., 2008; Segovia et al., 2016). Studies on species richness of these organisms are practically non-existent due to difficulties still existent in the taxonomic identification of the species (Auer and Arndt, 2001; Debastiani et al., 2016). According to Debastiani et al. (2016), there are few studies on the longitudinal variation of protist species richness and density in urban streams.

We aimed to investigate spatial and temporal scales, abundance, and factors that structure the communities of protozoans in a tropical urban stream. Furthermore, we characterized the limnology of this stream, providing information on water quality through the evaluation of Indexes of Water Quality and Trophic State.

\section{Material and Methods}

\subsection{Study area}

Samples were taken in the Conservation Unit of Parque do Cinquentenário $\left(23^{\circ} 22^{\prime} \mathrm{S}\right.$; $\left.51^{\circ} 56^{\prime} \mathrm{W}\right)$, in the city of Maringá, Paraná State, Brazil, which possesses an area of 18.38 ha. The park comprises part of the medium and low slopes of the Mandacaru stream, belonging to the Pirapó River watershed, comprising a larger area on its right margin and extending to the bottom of the stream valley. On the left margin, the park comprises only the valley bottom (Figure 1). This stream suffers the impacts caused by domestic and industrial effluents, especially in the Point 4.

The park presents no eroded sites. However, erosion is present in its surroundings, due to the absence of riparian forest. Anthropic action around the park is pronounced, with the presence of great quantity of construction debris and solid residuals (Paula and Ferreira, 2005).

\subsection{Field sampling}

Due to the stream extension, we established six equidistant sampling points along the longitudinal axis of the stream, with approximately 100 meters between 


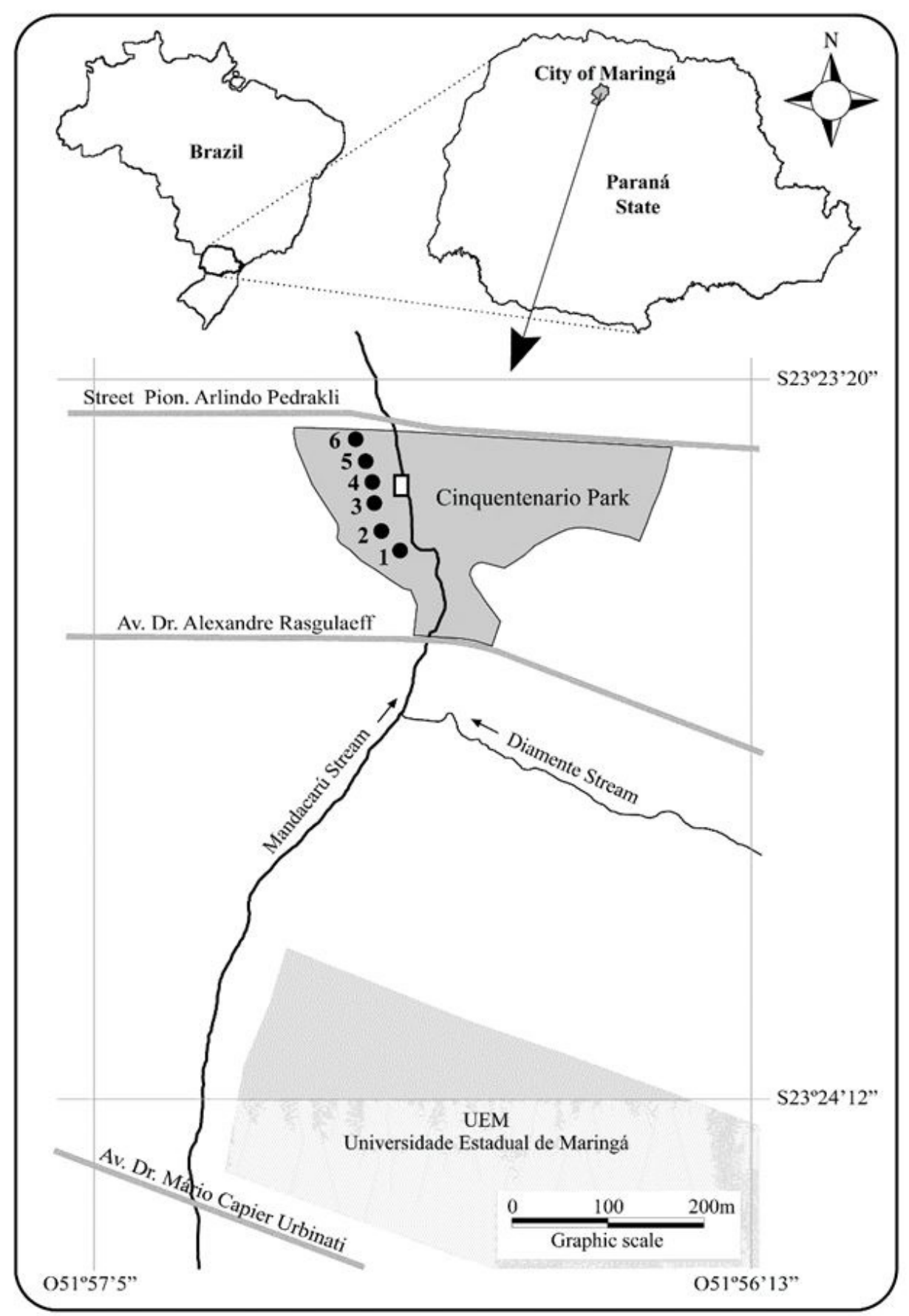

Figure 1. Parque Cinquentenário, anthropized forest fragment located in the city of Maringá, PR. The arrow indicates water flow direction; the rectangle indicates the direct sewage discharge in the stream.

each point. Sampling was conducted bimonthly, between April 2012 and April 2013. We calculated precipitation for each sampling period as the mean precipitation of the five days preceding the sample. The Main Climatologic Station of Maringá provided rainfall data.

We sampled ciliate (4 liters) and bacteria $(100 \mathrm{~mL})$ passing a polyethylene flask at the subsurface of the water column. For the testate amoebae, we used a graduated bucket to filter 200 liters of water by sample with a plankton net of $68 \mu \mathrm{m}$ of mash opening. Bacteria samples were fixed with a solution composed of formaldehyde, alkaline lugol and thiosulfate (Sherr and Sherr, 1993). Testate amoebae were preserved in formaldehyde at $4 \%$ buffered with calcium carbonate. Ciliate samples were 
kept alive to avoid cellular deformations resulting from the fixation process.

We used samples in sterile glass flasks kept under refrigeration to microbiological tests. We used the multiple-tube method to determine thermotolerant coliforms (APHA, 1985).

We measured electric conductivity $\left(\mathrm{S} . \mathrm{cm}^{-1}\right)$, dissolved oxygen $\left(\mathrm{mg} . \mathrm{L}^{-1}\right), \mathrm{pH}$, depth, and water temperature $\left({ }^{\circ} \mathrm{C}\right)$ in the field, with portable devices. Water samples were taken through the immersion of polyethylene flasks $30 \mathrm{~cm}$ under the water surface. These samples were used to analyze the following variables: Chemical Oxygen Demand (COD) (mg.L $\left.\mathrm{L}^{-1}\right)$, Biochemical Oxigen Demand (BOD) (mg.L $\mathrm{L}^{-1}$ ), chlorophyll- $a\left(\mu \mathrm{g} . \mathrm{L}^{-1}\right)$, total phosphorous $\left(\mathrm{mg} . \mathrm{L}^{-1}\right)$, total nitrogen (mg. $\left.\mathrm{L}^{-1}\right)$, turbidity (NTU), and total solids (mg. $\left.\mathrm{L}^{-1}\right)$.

\subsection{Laboratory analyses}

We stained samples with Rose Bengal and counted only the organisms that presented a colored protoplasm, assuming they were alive at the sampling moment. We withdrew six subsequent subsamples with a Hensen-Stempel pipete of $2.5 \mathrm{~mL}$ and analyzed in Sedgwick-Rafter chamber to make a quantitative analysis of samples. Individuals were withdrew from each sample and were assembled in slides with glycerin for posterior identification. We analyzed all samples with an optic microscope (Olympus). For the qualitative analysis, we assembled 10 slides to verify the occurrence of new species in each sample. Taxonomic identification of species was based on the following literature: Ogden and Hedley (1980), Velho et al. (1996), Velho and Lansac-Tôha (1996) and Lansac-Tôha et al. $(2008,2014)$. Taxonomic framework of testate amoebae was based on that proposed by Adl et al. (2005). Thus, organisms were grouped in Arcellinida Kent, 1880 (Amoebozoa, Testacealobosia).

Ciliate density was estimated in vivo in an optic microscope Olympus CX41, within six hours of sampling, in increases of $100 \mathrm{x}$ and $400 \mathrm{x}$. We counted 10 slides per sample, and expressed total density in individuals. $\mathrm{L}^{-1}$. Identification was based on specialized literature (Foissner and Berger, 1996; Foissner et al., 1999). Taxonomic classification was performed according to Lynn (2008).

To determine the density of bacterioplankton, we filtered subsamples of $1 \mathrm{~mL}$ of water in a black filter Nucleopore/Watchman with $0.2 \mu \mathrm{m}$ of pore opening stained with $1 \mathrm{~mL}$ of DAPI fluorochrome (4.6-diamidin-2-phenylindole) at $0.1 \%$ during five minutes in the dark. We quantified bacteria in an increase of $1000 \mathrm{x}$ in epifluorescent microscope (Bjørnsen, 1986). Total density was expressed in cells. $\mathrm{mL}^{-1}$.

We determined thermotolerant coliforms according to methods specified in "Standard Method for the Examination of Water and Wastewater", edited by the "American Public Health Association" (APHA, 2006). Values obtained from the analysis were compared with values recommended by the CONAMA Resolution 357/2005 (Article 34) (Brasil, 2005) and by the São Paulo State Decree No 8.468/1976 (Article 18), with the aim of verifying if the parameters are within patterns acceptable by the mentioned legislations.
Effluents of any nature may only be discharged into the inland or coastal waters, surface or underground, when they meet the values stipulated by these decrees.

Concentrations of coliforms, COD, BOD, total phosphorous, total nitrogen, and total solids were determined by the Labsam laboratory - Laboratory of Environmental Quality, according to APHA (2006). Concentrations of chlorophyll-a $\left(\mu \mathrm{g} . \mathrm{L}^{-1}\right)$ were determined by the Laboratory of Basic Limnology of Nupelia/UEM, according to Golterman et al. (1978).

\subsection{Data analysis}

We summarized data from physical and chemical water variables as water temperature $\left({ }^{\circ} \mathrm{C}\right)$, dissolved oxygen $\left(\mathrm{mg} . \mathrm{L}^{-1}\right), \mathrm{pH}$, electric conductivity $\left(\mathrm{S} . \mathrm{cm}^{-1}\right)$, turbidity (NTU), phosphorous (mg. $\left.\mathrm{L}^{-1}\right)$, nitrogen $\left(\mathrm{mg} . \mathrm{L}^{-1}\right)$, total solids (mg. $\mathrm{L}^{-1}$ ), Biochemical Oxygen Demand (mg. $\mathrm{L}^{-1}$ ), and Chemical Oxygen Demand (mg. $\left.\mathrm{L}^{-1}\right)$ in an Analysis of Principal Components (PCA). We used the Broken-Stick criterion (Jackson, 1993) to select the significant PCA axes. Before the analysis, variables were $\ln (\mathrm{x}+1)$ transformed, except for $\mathrm{pH}$. This analysis was realized in the software PC-ORD, version 4.01 (McCune and Mefford, 1999).

Calculation of the Water Quality Index (WQI), composed by nine parameters of water quality (dissolved oxygen, thermotolerant coliforms, $\mathrm{pH}, \mathrm{BOD}$, water temperature, total nitrogen, total phosphorous, turbidity, and total solids), was made according to the Formula 1 (ANA, 2004),

$W Q I=\sum_{i=1}^{n} q i^{w i}$

where: WQI = Water Quality Index, varying between 0 and 100; qi = quality of the $\mathrm{i}$-th parameter, varying between 0 and 100, obtained from the respective quality graphic, in function of its concentration or measurement (result of the analysis); wi = weight correspondent to the i-th parameter fixed in function of its importance for the global quality importance, that is, a number between 0 and 1 , so that: being $n$ the number of parameters considered in the WQI calculation.

WQI calculation required a normalization, where each parameter was transformed in a scale between 0 and $100 \%$, with $100 \%$ representing the best water quality. Then, we applied weights to each parameter according to their importance (Table 1) (Pesce and Wunderlin, 2000).

We classified water from each sampled point in the stream with WQI according to CETESB (2008) in: Very good (WQI between 80 and 100), Good (WQI between 52 and 79), Regular (WQI between 37 and 51), Bad (WQI between 20 and 36), and Very bad (WQI between 0 and 19).

We also calculated the Trophic State Index for phosphorous - TSI (TP) (modified by Lamparelli, 2004), established for lotic environments, according to the Equation 2:

$T S I(T P)=10 x(6-((0,42-0,36 x(\ln T P)) / \ln 2))-20$

where: $\mathrm{TP}=$ total phosphorous concentration measured at water surface, in mg. $\mathrm{L}^{-1}$; $\ln =$ natural logarithm. 
To interpret the results, sampled points were classified according to limits established to the different trophic classes in rivers, according to Lamparelli (2004).

Species composition was considered only for the communities of testate amoebae and ciliates. Testate protozoans were grouped by Family and ciliates by Order.

We calculated frequency of occurrence of each species (Constancy Index) through the percentage of samples in which the species occurred, according to the expression: $\mathrm{c}=\mathrm{n} \times 100 / \mathrm{N}$, where $\mathrm{n}$ is the number of samples in which the species occurred and $\mathrm{N}$ is the total number of samples. Species were classified as to occurrence in constant (present in more than $50 \%$ of samples), accessory (between $25 \%$ and $50 \%$ of samples), and rare (occurring in less than $25 \%$ of samples) (Dajoz, 1973).

Furthermore, we calculated species richness (for testate amoebae and ciliates) and mean density (for the three protoplankton groups) for each sample point and hydrological period and compared density among groups. To evidence statistical differences among richness and density data, we realized variance analyses (ANOVA) with the software Statistica 7.1 (Statsoft, 2005), considering as significant $p$ values larger than 0.05 .

We characterized species dominance for testate amoebae and ciliates according to Lobo and Leighton (1986):

a) dominant species those that occurred in densities superior to $50 \%$ of total sample density;

b) abundant species those that occurred in densities superior to mean sample density.

We used a Redundancy Analysis (RDA) to verify the influence of environmental variables, bacteria, and chlorophyll in the protozoan community (testate amoebae and ciliates). This method combines Linear Regression and Principal Component Analysis (PCA), and is an extension of Regression Analysis to model multivariate data (Legendre and Legendre, 1998). For this, we used a matrix with density values of the distinct protozoan groups and a matrix with abiotic variables (water temperature, $\mathrm{pH}$, electric conductivity, dissolved oxygen, total phosphorous, total nitrogen, chemical oxygen demand,

Table 1. Relative weight of each limnological variable used in WQI calculation.

\begin{tabular}{lc}
\hline \multicolumn{1}{c}{ Variables } & $\begin{array}{c}\text { Relative } \\
\text { weight (wi) }\end{array}$ \\
\hline Thermotolerant coliforms (TC) & 0.16 \\
Biochemical oxygen demand (BOD) & 0.11 \\
Total phosphorous (TP) & 0.10 \\
Total nitrogen (TN) & 0.10 \\
Dissolved oxygen (DO) & 0.17 \\
Potential Hydrogenion (pH) & 0.11 \\
Total solids (TS) & 0.7 \\
Turbidity (Turb) & 0.08 \\
Water temperature (Temp) & 0.10 \\
\hline
\end{tabular}

and biochemical oxygen demand), total chlorophyll, and bacterioplankton density. Biotic and abiotic data were previously logarithmized, except for $\mathrm{pH}$. These analysis were realized in software R, version 3.0.1 (R Core Team, 2013), with the package vegan.

\section{Results}

\subsection{Pluviometric precipitation}

To evaluate mean precipitation during the study period we considered data obtained in the five days prior to sampling date. Thus, We considered samples realized in April, July, and December 2012 as dry period, and samples made in September 2012 and February and April 2013 as rain period (Figure 2 ).

\subsection{Limnological variables}

Results of the Principal Component Analysis (PCA) evidenced a higher temporal than spatial segregation in sampling units. The first and second PCA axes were significant $(p<0.05)$. Thus, samples in the rainy period were negatively correlated to the first PCA axis, and were characterized by turbidity, COD, BOD, oxygen, and $\mathrm{pH}$. Samples in the dry period were positively correlated to this axis, presenting higher values of nitrogen, total solids, conductivity, and temperature (Figure 3 ).

\subsection{Water Quality Index (WQI)}

We evaluated water quality index (WQI) mainly as acceptable during the entire study period in the majority of sampled points (mean between 37.79 and 50.73). Water quality was considered good in some points (mean between 58.17 and 66.51), and considered bad in points 4 and 6 during the first and fourth sampling campaigns (mean between 30.55 and 32.57).

Results obtained for each parameter composing WQI are presented in Table 2.

\subsection{Trophic State Index (TSI)}

We classified the trophic state of streams according to CETESB, 2004 (Table 3). In most of the sampled points the streams were considered mesotrophic (mean

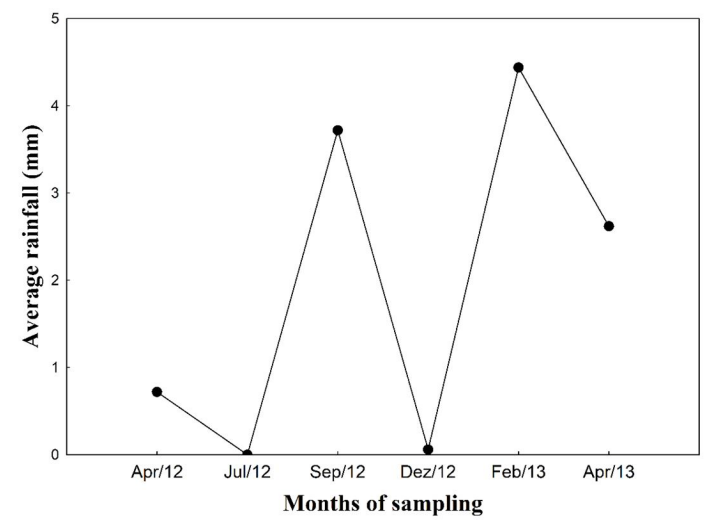

Figure 2. Precipitation mean during the five days prior to each sampling date. 
Table 2. Minimum (Min), maximum (Max), and mean values of limnological variables used for the WQI calculation.

\begin{tabular}{|c|c|c|c|c|c|c|c|}
\hline \multirow{2}{*}{ Variables } & \multirow{2}{*}{ Unit } & \multicolumn{3}{|c|}{ Rainy } & \multicolumn{3}{|c|}{ Dry } \\
\hline & & Mean & Min & Max & Mean & Min & Max \\
\hline Water temperature & ${ }^{\circ} \mathrm{C}$ & 21.7 & 18.2 & 25 & 22.4 & 19.3 & 24.4 \\
\hline Dissolved oxygen & $\mathrm{mg} \cdot \mathrm{L}^{-1}$ & 6.44 & 5.41 & 7.04 & 6.87 & 4.52 & 9.53 \\
\hline BOD & $\mathrm{mg} \cdot \mathrm{L}^{-1}$ & 5.99 & 3.25 & 8.81 & 5.0 & 1.1 & 23.6 \\
\hline $\mathrm{pH}$ & & 6.88 & 4.75 & 7.4 & 6.75 & 5.15 & 8.36 \\
\hline Thermotolerant coliforms & UFC. $100 \mathrm{~mL}^{-1}$ & $1.87 \times 10^{4}$ & $4 \times 10^{2}$ & $27 \times 10^{4}$ & $9.68 \times 10^{4}$ & $4 \times 10^{2}$ & $9 \times 10^{4}$ \\
\hline Total solids & mg. $\mathrm{L}^{-1}$ & 199 & 164 & 234 & 347.4 & 138 & 628 \\
\hline Total nitrogen & $\mathrm{mg} \cdot \mathrm{L}^{-1}$ & 3.02 & 2.09 & 3.6 & 5.13 & 2.96 & 11.35 \\
\hline Total phosphorous & $\mu \mathrm{g} . \mathrm{L}^{-1}$ & 0.05 & 0.01 & 0.1 & 0.25 & 0.02 & 1.2 \\
\hline Turbidity & NTU & 9.25 & 4.23 & 15.8 & 2.34 & 1 & 4.34 \\
\hline
\end{tabular}

Table 3. Classification of trophic state used for lotic environments.

\begin{tabular}{lc}
\hline \multicolumn{1}{c}{ Trophic state } & $\begin{array}{c}\text { Total phosphorous } \\
\left(\mathbf{m g}^{-3}\right)\end{array}$ \\
\hline Ultraoligotrophic & $\mathrm{P} \leq 13$ \\
Oligotrophic & $13 \mathrm{P} \leq 35$ \\
Mesotrophic & $35 \mathrm{P} \leq 137$ \\
Eutrophic & $137 \mathrm{P} \leq 296$ \\
Supereutrophic & $296 \mathrm{P} \leq 640$ \\
Hypereutrophic & $640 \mathrm{P}$ \\
\hline
\end{tabular}

Source: CETESB (2004).

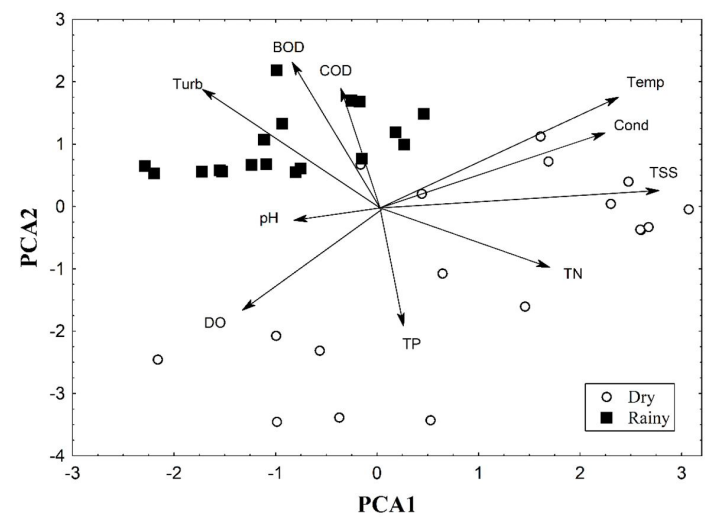

Figure 3. Dispersion of the scores of sampling points along the first two PCA axes (Turb: turbidity; COD = chemical oxygen demand; BOD = biochemical oxygen demand; Temp = temperature; Cond. = electric conductivity; $\mathrm{TSS}=$ total solids; $\mathrm{TN}=$ total nitrogen; $\mathrm{TP}=$ total phosphorous; DO = dissolved oxygen).

between 53.09 and 58.35). During the second sampling, two points were classified as eutrophic (points 1 and 6) with values varying between 59.96 and 60.61. Moreover, point 4 was considered supereutrophic during the fourth sampling (64.05) and as hipereutrophic in the second sampling (70.76).

\subsection{Species composition}

We identified 19 taxa of testate amoebae, belonging to six families, being Difflugiidae (six taxa), Centropyxidae (five taxa), and Arcellidae (four taxa) those with more species.
Although Difflugiidae is the family with most species, all taxa in this family were classified as rare by frequency of occurrence of each species (Constancy Index). Centropyxidae and Arcellidae were the only families that presented constant species in most of the sampled points, highlighting Arcella discoides and Centropyxis aculeata (Table 4).

We identified 71 infrageneric taxa of ciliates, distributed in 12 orders. Peniculida was the most representative order (19 taxa), followed by Euplotida (12 taxa). Among ciliates, we did not identify any constant taxa, and most of them were classified as rare. Only in Peniculida, Euplotida, Peritrichida, Pleurostomatida, Philasterida, and Haptorida we registered accessory species (Table 4).

\subsection{Species richness}

Of the total protozoan richness (90 taxa), 71 were ciliates and 19 testate amoebae (Figure 4; Table 4). Total richness was higher in the rainy period. Ciliates presented a higher number of taxa in both studied periods (dry and rainy). However, ciliate richness was significantly higher in the rainy period $(\mathrm{F}=11.54 ; p=0.001)$. Testate richness presented no significant difference between periods $(\mathrm{F}=4.11 ; p=0.0503)$.

Ciliate richness was, in general, proportionately higher than testate richness in sampled points (Figure 5). Considering each group, testate amoebae presented higher richness in points 1,3 and 5, and ciliates in points 1,5 , and 6 (Figure 5).

Testate density was significantly higher in dry periods (April, July, and December 2012) ( $\mathrm{F}=4.35 ; p=0.04)$, with higher values registered in April. The smallest density was registered in February 2013, rainy period (Figure 6).

Considering sampling points, we observed the same pattern as to the studied periods (higher values during dry periods). Higher values were found in point 5, during drought. In the rainy period, values were not higher than 200 ind. $\mathrm{m}^{-3}$, except in point 3 (Figure 7).

Contrary to the observed for testate amoebae, higher ciliate density was observed during the rainy season, especially in the third campaign (Figure 8). This difference in ciliate density between periods was considered significant $(\mathrm{F}=8.7 ; p=0.005)$.

There was a representative difference in ciliate density during the rainy period in comparison with the dry period, when evaluating the distribution of this attribute among sampling points (Figure 9). The dry period was kept uniform and with lower densities in the sampling points. 
Table 4. List of testate amoebae species (grouped by family) and ciliate species (grouped by order) and frequencies of occurrence in each sampling point, according to the Dajoz index $(\mathrm{xxx}=$ constant; $\mathrm{xx}=$ accessory; $\mathrm{x}=$ rare $)$ and according to the dominance index (light grey = abundant; dark grey = dominant).

\section{TESTATE AMOEBAE}

SAMPLING POINTS

ARCELLIDAE Ehrenberg, 1830

Arcella discoides Ehrenberg, 1843

Arcella hemisphaerica Perty, 1852

Arcella megastoma Pénard, 1902

Arcella vulgaris Ehrenberg, 1830

CENTROPYXIDAE Deflandre, 1953

Centropyxis aculeata (Ehrenberg, 1838)

Centropyxis constricta (Ehrenberg, 1838)

Centropyxis discoides (Pénard, 1890)

Centropyxis ecornis (Ehrenberg, 1841)

Centropyxis hirsuta Deflandre, 1929

DIFLLUGIIDAE Awerintzew, 1906

Mediolus corona (Wallich, 1854)

Difflugia gramen Pénard, 1902

Difflugia lithophila Pénard, 1902

Difflugia lobostoma Leidy, 1879

Difflugia oblonga Ehrenberg, 1838

Difflugia stellastoma Vucetich, 1989

TRIGONOPYXIDAE Loeblich and Tappan, 1964

Cyclopyxis impressa (Daday, 1905)

Cyclopyxis kahli Deflandre, 1929

LESQUEREUSIIDAE Jung, 1942

Lesquereusia spirallis (Ehrenberg, 1840)

PLAGIOPYXIDAE Bonnet \& Thomas 1960

Plagiopyxis callida Pénard, 1910

\section{CILIATES}

\begin{tabular}{cccccc}
\multicolumn{6}{c}{ SAMPLING POINTS } \\
\hline $\mathbf{1}$ & $\mathbf{2}$ & $\mathbf{3}$ & $\mathbf{4}$ & $\mathbf{5}$ & $\mathbf{6}$ \\
$\mathbf{x x}$ & $\mathbf{x x}$ & $\mathbf{x x x}$ & $\mathbf{x x}$ & $\mathbf{x x x}$ & $\mathbf{x x x}$ \\
$\mathbf{x x}$ & $\mathbf{x}$ & $\mathbf{x}$ & $\mathbf{x x}$ & $\mathbf{x x}$ & $\mathbf{x}$ \\
$\mathbf{x}$ & - & $\mathbf{x}$ & - & $\mathbf{x}$ & $\mathbf{x}$ \\
& & & & & \\
$\mathbf{x x x}$ & $\mathbf{x x x}$ & $\mathbf{x x x}$ & $\mathbf{x x x}$ & $\mathbf{x x x}$ & $\mathbf{x x x}$ \\
$\mathbf{x}$ & - & $\mathbf{x}$ & - & - & - \\
$\mathbf{x x x}$ & $\mathbf{x x}$ & $\mathbf{x x x}$ & $\mathbf{x x}$ & - & $\mathbf{x x}$ \\
$\mathbf{x x}$ & $\mathbf{x x x}$ & $\mathbf{x x x}$ & $\mathbf{x x}$ & $\mathbf{x x}$ & $\mathbf{x x}$ \\
$\mathbf{x x}$ & $\mathbf{x}$ & $\mathbf{x x}$ & $\mathbf{x}$ & $\mathbf{x x}$ & -
\end{tabular}

\section{COLPODIDA}

Colpoda magna Gruber, 1880

Colpoda steinii Maupas, 1883

Colpoda sp.

Cyrtolophosis mucicola Stokes, 1885

\section{CHLAMYDODONTIDA}

Chilodonella uncinata (Ehrenberg, 1838)

Trithigmostoma sp.

\section{HETEROTRICHIDA}

Blepharisma sp.

Stentor sp.

\section{PENICULIDA}

Colpidium colpoda Gannerr \& Foissner, 1989

Dexiotricha granulosa Foissner, Berger \& Kohmann, 1994

Disematostoma tetraedricum Kalh, 1931

Disematostoma sp.

Epenardia myriophylli Corliss, 1971

Frontonia leucas Ehrenberg, 1838

Glaucoma reniforme (Schewiakoff, 1892)

Glaucoma scintillans (Ehrenberg, 1830)

Glaucoma sp.

Lembadium bullinum (Perty, 1852)

$\begin{array}{llllll}1 & 2 & 3 & 4 & 5 & 6\end{array}$

\begin{tabular}{cccccc}
- & $\mathbf{x}$ & - & - & $\mathbf{x}$ & $\mathbf{x}$ \\
- & $\mathbf{x}$ & - & - & - & - \\
- & $\mathbf{x}$ & $\mathbf{x}$ & - & - & - \\
- & - & - & - & - & $\mathbf{x}$ \\
- & - & - & $\mathbf{x}$ & - & - \\
- & - & - & $\mathbf{x}$ & - & $\mathbf{x}$ \\
& & & & & \\
- & - & - & $\mathbf{x}$ & $\mathbf{x}$ & $\mathbf{x}$ \\
- & - & $\mathbf{x}$ & - & $\mathbf{x}$ & - \\
& & & & & \\
- & $\mathbf{x}$ & - & $\mathbf{x}$ & - & - \\
- & - & - & $\mathbf{x}$ & - & - \\
- & - & - & $\mathbf{x}$ & - & - \\
- & - & - & - & $\mathbf{x}$ & - \\
$\mathbf{x}$ & $\mathbf{x}$ & - & - & $\mathbf{x}$ & - \\
$\mathbf{x}$ & - & - & - & - & - \\
$\mathbf{x}$ & $\mathbf{x}$ & $\mathbf{x}$ & $\mathbf{x}$ & $\mathbf{x}$ & - \\
- & - & $\mathbf{x x}$ & $\mathbf{x}$ & - & - \\
- & - & - & - & - & $\mathbf{x}$ \\
- & - & $\mathbf{x}$ & - & - & $\mathbf{x}$ \\
\hline & & & & &
\end{tabular}


Table 4. Continued...

\begin{tabular}{|c|c|c|c|c|c|c|}
\hline \multirow{2}{*}{ CILIATES } & \multicolumn{6}{|c|}{ SAMPLING POINTS } \\
\hline & 1 & 2 & 3 & 4 & 5 & 6 \\
\hline Lembadium lucens (Maskell, 1887) & $\mathbf{x}$ & - & - & - & - & $\mathbf{x}$ \\
\hline Ophyryoglena sp. & - & $\mathbf{x}$ & - & - & - & - \\
\hline Paramecium aurelia complex. & $\mathbf{x}$ & $\mathbf{x}$ & - & $\mathbf{x}$ & $\mathbf{x}$ & $\mathbf{x}$ \\
\hline Paramecium bursaria (Ehrenberg, 1831) & $\mathbf{x}$ & - & $\mathbf{x}$ & $\mathbf{x}$ & - & $\mathbf{x}$ \\
\hline Paramecium caudatum Ehrenberg, 1834 & - & $\mathbf{x}$ & $\mathbf{x}$ & - & $\mathbf{x}$ & $\mathbf{x}$ \\
\hline Stokesia vernalis Wenrich, 1929 & - & - & $\mathbf{x}$ & - & $\mathbf{x}$ & - \\
\hline Tetrahymena pyriformis (Ehrenberg, 1830) & $\mathbf{x}$ & $\mathbf{x x}$ & $\mathbf{x x}$ & - & $\mathbf{x}$ & $\mathbf{x}$ \\
\hline Tetrahymena sp. & - & - & $\mathbf{x}$ & $\mathbf{x}$ & - & - \\
\hline Urocentrum turbo (Mueller, 1786) & $\mathbf{x x}$ & - & $\mathbf{x}$ & $\mathbf{x}$ & $\mathbf{x x}$ & $\mathbf{x}$ \\
\hline \multicolumn{7}{|l|}{ EUPLOTIDA } \\
\hline Aspidisca cicada (Mueller, 1786) & - & $\mathbf{x x}$ & $\mathbf{x}$ & - & $\mathbf{x}$ & $\mathbf{x x}$ \\
\hline Aspidisca lynceus (Mueller, 1773) & $\mathbf{x}$ & $\mathbf{x}$ & $\mathbf{x x}$ & - & $\mathbf{x}$ & - \\
\hline Euplotes aediculatus Pierson, 1943 & - & $\mathbf{x x}$ & $\mathbf{x x}$ & $\mathbf{x}$ & - & $\mathbf{x}$ \\
\hline Euplotes eurystomus (Wrzesniowski, 1870) & - & $\mathbf{x x}$ & - & $\mathbf{x}$ & $\mathbf{x x}$ & $\mathbf{x x}$ \\
\hline Euplotes moebiusi Kahl, 1932 & $\mathbf{x}$ & - & - & - & - & $\mathbf{x}$ \\
\hline Hypotrichida sp. & - & - & - & $\mathbf{x x}$ & - & $\mathbf{x}$ \\
\hline Hypotrichium sp. & - & - & $\mathbf{x}$ & - & - & $\mathbf{x}$ \\
\hline Holosticha sp. & - & - & - & - & - & $\mathbf{x}$ \\
\hline Oxytricha saprobia Kahl, 1932 & $\mathbf{x}$ & - & - & - & - & - \\
\hline Oxytricha similis Engelmann, 1862 & $\mathbf{x}$ & - & - & - & - & - \\
\hline Oxytricha sp. & $\mathbf{x}$ & - & - & $\mathbf{x}$ & $\mathbf{x}$ & - \\
\hline Stichotricha secunda Perty, 1849 & - & - & $\mathbf{x}$ & - & - & - \\
\hline \multicolumn{7}{|l|}{ OLIGOTRICHIDA } \\
\hline Halteria grandinella (Mueller, 1773) & $\mathbf{x}$ & $\mathbf{x}$ & - & - & $\mathbf{x}$ & $\mathbf{x}$ \\
\hline Limnostrombidium sp. & $\mathbf{x}$ & $\mathbf{x}$ & $\mathbf{x}$ & $\mathbf{x}$ & - & - \\
\hline \multicolumn{7}{|l|}{ PERITRICHIDA } \\
\hline Carchesium polypinum Linnaeus, 1758 & - & $\mathbf{x}$ & - & - & - & $\mathbf{x}$ \\
\hline Epistyllis hentcheli Kahl, 1935 & - & $\mathbf{x}$ & - & - & - & - \\
\hline Epistyllis sp. & $\mathbf{x x}$ & - & - & - & $\mathbf{x x}$ & $\mathbf{x}$ \\
\hline Vorticella aquadulcis complex & $\mathbf{x x}$ & $\mathbf{x}$ & $\mathbf{x}$ & $\mathbf{x}$ & $\mathbf{x x}$ & $\mathbf{x x}$ \\
\hline Vorticella campanula Ehrenberg, 1831 & - & $\mathbf{x x}$ & $\mathbf{x}$ & $\mathbf{x}$ & $\mathbf{x x}$ & $\mathbf{x}$ \\
\hline Vorticella convallaria complex & $\mathbf{x x}$ & - & - & $\mathbf{x x}$ & $\mathbf{x x}$ & $\mathbf{x}$ \\
\hline Vorticella mayeri Faure-Fremiet, 1920 & - & $\mathbf{x}$ & $\mathbf{x}$ & - & - & - \\
\hline Vorticella sp. & - & - & $\mathbf{x}$ & $\mathbf{x}$ & - & - \\
\hline \multicolumn{7}{|l|}{ PLEUROSTOMATIDA } \\
\hline Acineria uncinata Tucolesco, 1962 & - & - & - & $\mathbf{x}$ & $\mathbf{x}$ & - \\
\hline Amphileptus pleurosigma (Stokes, 1884) & - & $\mathrm{x}$ & - & - & - & $\mathrm{xx}$ \\
\hline Amphileptus procerus (Penard, 1922) & $\mathrm{x}$ & - & $\mathrm{x}$ & - & - & - \\
\hline Litonotus alpestris Foissner, 1978 & $\mathrm{x}$ & - & - & - & $\mathbf{x}$ & $\mathrm{x}$ \\
\hline Litonotus crystallinus (Vuxanovici, 1960) & - & $\mathrm{x}$ & - & - & $\mathbf{x}$ & - \\
\hline Litonotus lamella (Mueller, 1773) & $\mathrm{x}$ & - & - & - & - & - \\
\hline \multicolumn{7}{|l|}{ PRORODONTIDA } \\
\hline Balaniom planctonicum (Foissner et al., 1990) & - & - & $\mathbf{x}$ & - & - & - \\
\hline Coleps spetai Foissner, 1984 & - & - & $\mathbf{x}$ & - & - & - \\
\hline Holophrya discolor Ehrenberg, 1833 & - & - & - & - & $\mathbf{x}$ & - \\
\hline \multicolumn{7}{|l|}{ PHILASTERIDA } \\
\hline Cinetochilum margaritaceum (Ehrenberg, 1831) & $\mathbf{x x}$ & $\mathbf{x}$ & $\mathbf{x x}$ & - & $\mathbf{x}$ & $\mathbf{x x}$ \\
\hline Cyclidium glaucoma Mueller, 1773 & $\mathbf{x x}$ & $\mathbf{x x}$ & $\mathbf{x x}$ & $\mathbf{x x}$ & $\mathbf{x}$ & $\mathbf{x}$ \\
\hline Cyclidium $S P$ & $\mathbf{x}$ & - & - & $\mathbf{x}$ & $\mathbf{x x}$ & $\mathbf{x}$ \\
\hline
\end{tabular}


Table 4. Continued...

\begin{tabular}{|c|c|c|c|c|c|c|}
\hline \multirow{2}{*}{ CILIATES } & \multicolumn{6}{|c|}{ SAMPLING POINTS } \\
\hline & 1 & 2 & 3 & 4 & 5 & 6 \\
\hline Dexiotrichides centralis (Stokes, 1885) & $\mathbf{x}$ & - & - & - & - & - \\
\hline Sathophilus muscorum (Kahl, 1931) & $\mathbf{x}$ & - & - & - & - & - \\
\hline SUCTORIDA & & & & & & \\
\hline Podaphyra sp. & - & - & - & - & $\mathbf{x}$ & - \\
\hline HAPTORIDA & & & & & & \\
\hline Balantidium pellucidum Eberhard, 1862 & - & - & - & - & $\mathbf{x}$ & $\mathbf{x}$ \\
\hline Campanella sp. & $\mathrm{x}$ & $\mathrm{x}$ & - & - & - & - \\
\hline Cyclotrichium viride Gajewskaja, 1933 & - & - & - & - & $\mathbf{x}$ & - \\
\hline Dexiostoma campylum (Stokes, 1885) & $\mathrm{x}$ & - & $\mathbf{x}$ & - & $\mathbf{x}$ & $\mathbf{x}$ \\
\hline Philasterides armatus Kahl, 1931 & - & - & $\mathbf{x}$ & - & $\mathbf{x}$ & $\mathbf{x x}$ \\
\hline Monodinium balbiani Fabre-Domergue, 1888 & - & - & $\mathbf{x}$ & - & $\mathbf{x}$ & $\mathbf{x x}$ \\
\hline Platynematum sociale (Penard, 1922) & - & - & - & - & - & $\mathbf{x}$ \\
\hline
\end{tabular}

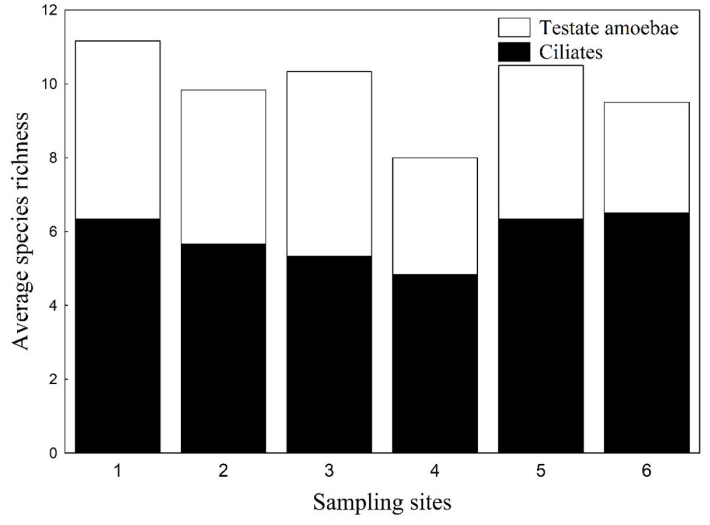

Figure 4. Total species richness estimated for testate amoebae and ciliates in sampled points during dry and rainy periods.

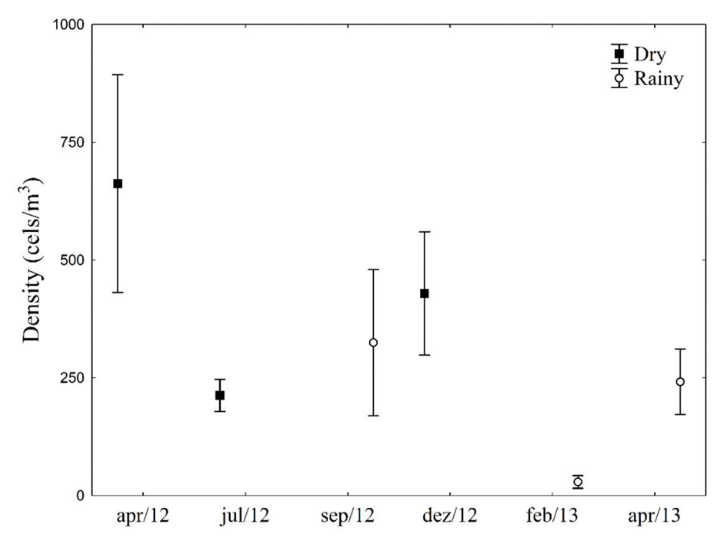

Figure 5. Average of the species richness of testate amoebae and ciliates in sampling sites.

\subsection{Species dominance}

For testate amoebae, Arcella discoides was considered abundant in all sampling points, and Centropyxis aculeata was classified as dominant in points 2 and 3 and abundant in all the other points (Table 4).

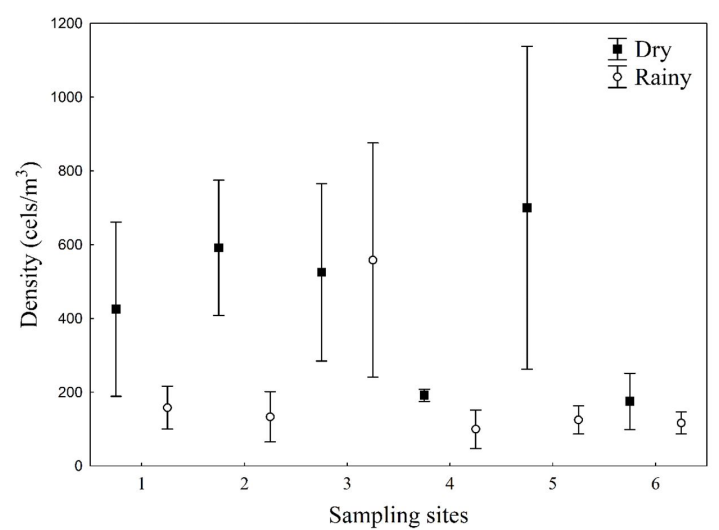

Figure 6. Mean and standard error of testate amoebae density in different hydrological periods.

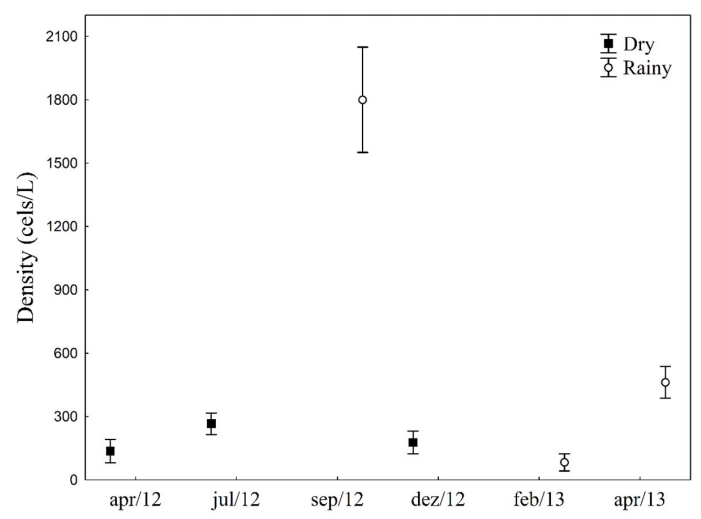

Figure 7. Mean and standard error of testate density in each hydrological period and sampling point.

For ciliates, we registered no dominant species. However, many taxa were classified as abundant, highlighting species of orders Peritrichida, especially Vorticella aquadulcis and $V$. convalaria, and Philasterida, especially Cyclidium glaucoma (Table 4). 


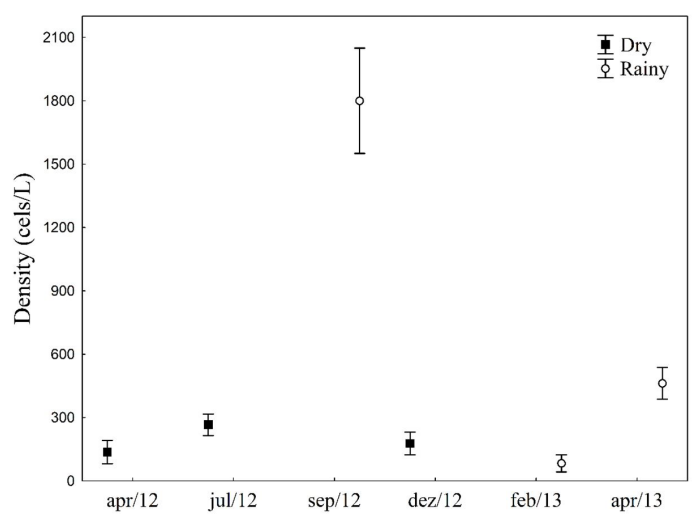

Figure 8. Mean and standard error of ciliate density in different hydrological periods.

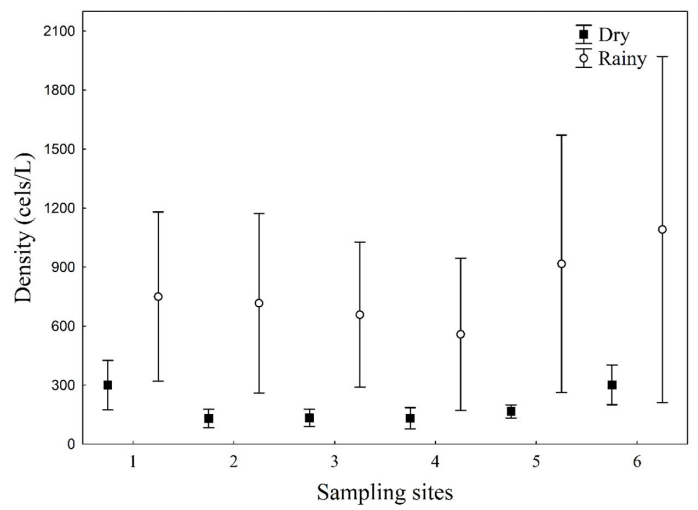

Figure 9. Mean and standard error of ciliate density in each hydrological period and sampling point.

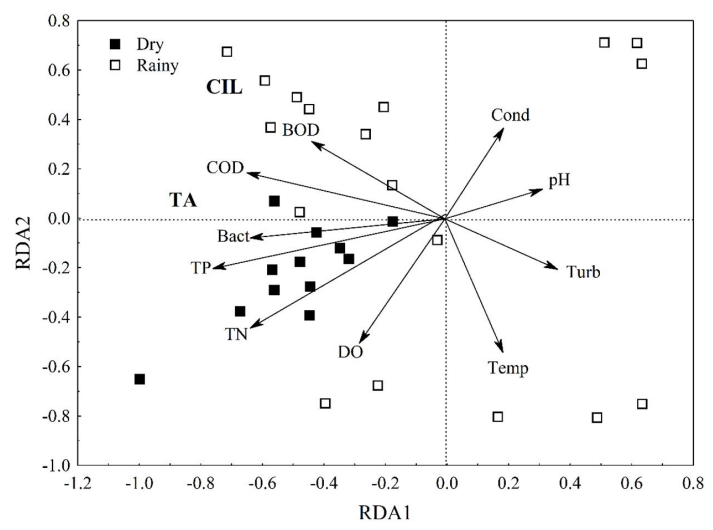

Figure 10. Ordination diagram for the first two axes of the Redundancy Analysis, with scores of the stream according to abiotic variables (Cond = electric conductivity; Temp = temperature; Turb = turbidity; DO = dissolved oxygen; $\mathrm{TN}=$ Total Nitrogen; $\mathrm{TP}=$ Total Phosphorous; BOD $=$ Biochemical Oxygen Demand COD = Chemical Oxygen Demand), biotic (Bact $=$ bacterioplankton), and protozoan $(\mathrm{TA}=$ testate amoebae; $\mathrm{CIL}=$ ciliates $)$.

\subsection{Effect of environmental heterogeneity on protozoan communities}

According to the RDA, environmental variables explained $41 \%$ of variability in abundance data of the three protozoan groups, and an ANOVA evidenced significance in both axes (axis $1=0.005$; axis $2=0.04$ ).

Results of the RDA showed, in general, the same pattern of distribution of the sampling units as described in the PCA. Thus, we observed also in this analysis a higher temporal (between hydrological periods) than spatial segregation (among sampling points) (Figure 10).

According to this analysis, samples of the dry period were characterized by higher values of bacteria density and concentrations of total phosphorous, and total nitrogen (Figure 10). On the other hand, ciliates presented higher abundance values in some of the months characterized by higher precipitation and where higher values of BOD and COD were found (Figure 10). Testate amoebae also presented higher abundances in conditions of higher system productivity (higher nutrient concentration, bacteria, $\mathrm{BOD}$, and COD), although higher density values were not observed in the rainy season (Figure 10).

\section{Discussion}

\subsection{Limnological variables, WQI and TSI}

According to parameters established by CETESB (2008), WQI results must be interpreted in relation to the individual behavior of each of the parameters that compose it. This is important especially when one or more of them is distorting the WQI results, due to their concentration and dynamics in the water body. This index may mask relevant information on the parameters due to its reductionist character. This evaluation is important, as it allows a more realistic diagnostic of water quality. The huge amount of waste that is generated in cities can reach streams and rivers when carried by rainwater and thus carry large amounts of inorganic and dissolved organic matter, which can make the water unsuitable for direct and indirect use (Ntengwe, 2006; Harfuch et al., 2019).

A large part of the obtained BOD values surpass the limit of $5 \mathrm{mg} . \mathrm{L}^{-1}$ established by the CONAMA resolution 357/05 (Brasil, 2005) for class 2 freshwater. The mean varied between 5.23 and $23.6 \mathrm{mg} . \mathrm{L}^{-1}$, with higher values found in points located near wastewater discharge made directly in the stream. The increase in BOD in water bodies may be a consequence of sewage discharge, which causes an increase in organic matter and, consequently, decrease dissolved oxygen through oxidation (CETESB, 2009).

The discharge of organic matter into the stream is generally higher in rainy periods, due to leakage. Although in these periods there is also a higher dilution of organic matter due to larger water volume, the organic matter discharge results in BOD increase. Accordingly, our PCA indicated a positive correlation between BOD and the rainy period.

For turbidity, there was no difference between sampled points, and all of them were in accordance to the CONAMA 
resolution (Brasil, 2005) that establishes $100 \mathrm{UNT}$ as maximum limit to type 2 freshwater. However, we highlight that the higher values were obtained in the rainy period, as evidenced by the positive correlation between turbidity and this hydrological period. Othes studies also have pointed that turbidity tends to increase when there is flow increase (Fritzsons et al., 2003; Harfuch et al., 2019). With precipitation, erosion and leaching become common around rivers and streams, favoring the dispersion of suspended particles in the water.

The maximum value of total solids is of $500 \mathrm{mg} . \mathrm{L}^{-1}$ according to the CONAMA resolution (Brasil, 2005). Most of our sampled points presented values lower than this limit, except for the first sample, in which we verified values ranging from 503 to $628 \mathrm{mg} . \mathrm{L}^{-1}$. The quantity of total solids in rivers, lakes, and streams indicates their degree of pollution so that the higher the concentration, the higher is the pollution (Almeida and Schwarzbold, 2003). Total solids may be from natural sources, sewage discharges, urban outlets or industrial waste discharges (Ntengwe, 2006).

The highest value of total phosphorous allowed by the resolution to lotic environments is of $0.1 \mathrm{mg} . \mathrm{L}^{-1}$ (Brasil, 2005). At the Mandacaru stream, concentration of this variable in general surpasses this limit, with means ranging from 0.1 to $1.2 \mathrm{mg} . \mathrm{L}^{-1}$. We found the highest value of phosphorous at the point where there is sewage discharge at the stream (point 4 during the dry period). In turn, Harfuch et al. (2019) in studies carried out in other streams of the Pirapó River basin, Paraná State, did not find values of this variable above that stipulated by the resolution of Brasil (2005).

Phosphorous is considered the main responsible for eutrophication in aquatic ecosystems (Sperling, 2007). Naturally, it comes from rocks, decomposition and superficial rainwater leakage. Artificially, it comes from particles in the atmosphere, fertilizers, and domestic and industrial wastewater (Wetzel, 2001). According to Lamparelli (2004), mean nutrient concentrations, especially total phosphorous, are higher in lotic than in lentic systems.

The highest total nitrogen value allowed by Brasil (2005) in class 2 waters is 10 mg. $\mathrm{L}^{-1}$. However, nitrate concentrations above $5 \mathrm{mg} . \mathrm{L}^{-1}$ indicate inadequate sanitary conditions. A value surpassing this limits was observed only at the point were there is sewage direct discharge at the stream (Point 4 in the dry period).

One of the established criteria for water potability is $\mathrm{pH}$. According to the resolution (Brasil, 2005), $\mathrm{pH}$ values may vary between 6.0 and 9.0. In our study, most of the $\mathrm{pH}$ values were within the established limits. Only in some periods, at the points influenced by sewage discharge, we registered values under 6.0. $\mathrm{pH}$ may be influenced by organic matter quantity in the water. In acid organic compounds and at the decomposition of organic matter, there is carbon dioxide liberation, which makes the water acid (Marotta et al., 2008). Similar results were found in streams of the Pirapó River basin, Paraná State (Harfuch et al., 2019). Low $\mathrm{pH}$ values are often related to the arrival and transport of matter from acid soils adjacent to the water bodies (Marotta et al., 2008).

The minimum limit of dissolved oxygen according to Brasil (2005) is of $5 \mathrm{mg} \cdot \mathrm{L}^{-1}$. At the Mandacaru stream, concentrations were in general above this limit, with the value $4.52 \mathrm{mg} . \mathrm{L}^{-1}$ in only one sampling unit. Reduction in dissolved oxygen is one of the indicators of water pollution, due to its consumption by the decomposition of organic compounds (CETESB, 2010).

The chlorophyll-a limit established by the resolution is of $30 \mu \mathrm{g} . \mathrm{L}^{-1}$ (Brasil, 2005), with values above this limit indicating eutrophication of the aquatic system. At the Mandacaru stream, all values were below this limit. We highlight that, in lotic environments, flow velocity constitutes a limiting factor to the development of typical planktonic populations, resulting in low primary productivity and, consequently, in low chlorophyll concentrations (Reynolds and Descy, 1996).

A limit of 1,000 thermotolerant coliforms. $100 \mathrm{~mL}^{-1}$ of water is allowed according to Brasil (2005). At the Mandacaru stream, this limit was surpassed in almost all sampled points, with values varying between 1,500 and 270,000 coliforms. $100 \mathrm{~mL}^{-1}$ in the rainy period and dry period, respectively. In only one sample the values were below the established limit (between 400 and 800 coliforms. $100 \mathrm{~mL}^{-1}$ of water). These results differ from those obtained in streams of the Pirapó River basin, Paraná State (Harfuch et al., 2019). The presence of thermotolerant coliform bacteria is an indicator of pollution by sanitary wastewater and animal waste (CETESB, 2010).

There was practically no variation in temperature between sampled points in the studied period. This is probably because all samples were taken during the morning period.

The TSI was developed for reservoirs, but has been applied to rivers. It is however necessary to make a critical review of its application in lotic systems (Lamparelli, 2004). This same author, when comparing TSI classifications obtained through phosphorous with chlorophyll-a values, verified great inconsistency between them when applying this index in lotic systems.

Contrary to what is frequently registered for reservoirs, trophy classifications in rivers were little coherent in São Paulo State, using the conjugated index that includes total phosphorous and chlorophyll-a (Lamparelli, 2004). Thus, in rivers, where chlorophyll concentration is generally low (Reynolds and Descy, 1996), using indexes that include this variable seems little efficient. For this reason, we decided not to include this variable in the TSI calculations in this study. On the other hand, in urban lotic systems, subjected to anthropic action, phosphorous concentrations tend to be high (Lamparelli, 2004).

The mesotrophic conditions tending to eutrophy/hypertrophy found in the Mandacaru stream have been found in other lotic systems (Zanini et al., 2010). This increase in trophy verified in some sampling points and periods may be due to the discharge of domestic wastewater directly into the stream. 


\subsection{Composition and richness of testate amoebae and ciliates}

The most representative families of testate amoebae in this study, Difflugiidae, Centropyxidae, and Arcellidae, are also the most representative in other studies realized in Brazilian freshwater systems (Lansac-Tôha et al., 2007, 2008, 2014; Alves et al., 2012; Mansano et al., 2013, among others).

However, there are few studies with these protozoans in urban streams. Among these, we highlight the one developed in the region northwest of the State of São Paulo, where 21 taxa have been identified (Fulone et al., 2005). These authors attributed the low species number to the eutrophication process in the studied streams. Lippert (2013) realized a taxonomic survey, description, and illustration of testate protozoans in three anthropized first order streams in the State of Paraná. In this study, 22 taxa were identified, and the author concluded that the low species richness was associated to negative impacts of anthropic intervention on this community. Thus, the small number of species in the Mandacaru stream (19 taxa) corroborates with results of the authors cited above. This evidences the anthropic effect on these urban ecosystems, considering the sampling points presented, in general, TSI varying between mesotrophic and hipereutrophic.

Many studies realized in freshwater environments have registered a close association between testate amoebae and aquatic macrophytes (Alves et al., 2012). Thus, the absence of these plants may also explain the low species richness in the stream.

According to Margalef (1963), systems with strong environmental fluctuations remain in a low maturity state. In these systems, species with higher potential growth rates become frequent, reducing diversity and hindering an efficient mechanism of ecosystem homeostasis. According to this tendency, the evaluated stream presented, as highlighted above, a low specific richness of testate amoebae, with an elevated representativeness of only three species, who were constant in almost all sampled points. Thus, Centropyxis aculeate, C. ecornis, and Arcella discoides, evidenced in this study, are among those with larger geographic distribution in Brazil. They have been registered in different freshwater systems, in samplings of plankton, sediment, and macrophyte associated fauna (Velho et al., 2003; Lansac-Tôha et al., 2014).

According to Velho et al. (2003), the occurrence of testate amoebae is associated to the shape of their theca. Thus, the above-mentioned taxa are well distributed in the Mandacaru stream probably due to their flattened shape, common in most Arcellidae and Centropyxidae taxa. The flattened theca brings adaptive advantages to the organisms that inhabit lotic environments, as this shape makes individuals less susceptible to carriage by water flow.

Among ciliates, the orders with higher species richness in our study (Peniculida, Euplotida, and Peritrichida) have been registered as representative in rivers and streams (Madoni and Braghiroli, 2007). Species in these orders are considered, in general, as interstitial benthonic, but are frequently found at the pelagic zone of aquatic systems (Velho et al., 2013).

Studies on ciliate species richness in lotic systems have shown a variation from 20 to 200 registered species (Madoni, 2005; Madoni and Braghiroli, 2007; Dias et al., 2008; Debastiani et al., 2016). Thus, species richness in our study (71 taxa) may be considered as relatively high, as samples were taken in only one stream. A study realized in only one urban stream at southeast Brazil identified 22 taxa (Dias et al., 2008).

Although no ciliate species have been identified as constant, some of them were considered accessories, from which we highlight Cinetochilum margaritaceum and Cyclidium glaucoma, who have been registered as frequent and abundant taxa in many rivers and streams (Madoni and Braghiroli, 2007; Bagatini et al., 2013; Segovia et al., 2016). These species, belonging to the order Philasterida, are registered mainly in mesotrophic and eutrophic environments (Beaver and Crisman, 1989), which are the predominant conditions of the Mandacaru stream. On the other hand, some groups as Oligotrichida present low species richness in the studied stream, which may be associated to the fact that species from this order are mainly found in oligotrophic environments and are adapted to the plankton habit (Beaver and Crisman, 1989).

The larger species richness of ciliates and testate amoebae found in the rainy periods may be due to the carriage of species from the benthonic compartment and littoral region to the water column, due to increase in water flow caused by rains (Lampert and Sommer, 2007). In this sense, the water column may act as a collector of protozoa from all the stream compartments, mainly those small and shallow (Velho et al., 2013; Lansac-Tôha et al., 2014). Similar results have been found by Fulone et al. (2005) in streams from the southeast region of Brazil.

\subsection{Density of testate amoebae and ciliates}

Among testate amoebae, Arcella discoides (Arcellidae) and Centropyxis aculeata (Centropyxidae) were the only species abundant in all sampled points. Centropyxis aculeata, besides being abundant, was the only dominating species. The family Centropyxidae contains opportunistic species found in impacted fluvial systems (Patterson and Kumar, 2000). According to Patterson and Kumar, (2000), C. aculeata tolerates environments contaminated by industrial effluents. In one of our sampled points where this species is dominant (P3), there is direct discharge of domestic wastewater. The numeric dominance of this species among testate amoebae has also been verified in other aquatic systems in Brazil (Lippert, 2013; Lansac-Tôha et al., 2014, among others).

Various studies have evidenced that the presence of disturbances and the dominance of tolerant species to the detriment of more sensible species markedly change the structure and distribution of fish fauna (Jackson et al., 2001). Cunico et al. (2012) evidenced that environmental changes related to pollutants have influenced fish assemblage in urban streams, including the Mandacaru. These authors 
verified that variation on the fish community structure was related to the entrance of organic nutrients in these environments. We emphasize that these urban streams present an elevated pollution degree due to urban occupation (allotments and industries) and to the reception of diverse effluents as non-treated domestic sewage. Such conditions may explain the fact that only one testate species was dominant in the Mandacaru stream.

The family Difflugiidae presented the highest number of species among testate amoebae, but was not abundant in our study. This family is the most diversified among testate amoebae (Lansac-Tôha et al., 2014), presenting a high variety of shapes. However, its predominantly globed or elongated shape limits the development of large populations in systems with elevated flow velocity (Velho et al., 2003).

No ciliate species was considered dominant in the different sampled points of the stream. However, species in some Orders, especially Peniculida and Peritrichida, presented abundant species. These ciliate groups have been frequently registered as abundant in lotic systems (Kiss et al., 2009).

The higher ciliate densities found in the rainy period diverge from results of other studies that found higher densities of these protozoans in the dry period (Velho et al., 2005,2013 ) due to the dilution effect on these individuals during the rainy period. The pluvial regime, mainly in lotic systems, is an important factor that changes ciliate protists abundances, as demonstrated in other studies (Primc-Habdija et al., 1998). The ciliate community was composed in great part of species not truly planktonic in both hydrological periods. Thus, it is likely that, in the rainy period, a great quantity of individuals from species that inhabit the sediment and littoral vegetation, and even the inundated soil, is carried to the water column. In this sense, some non-planktonic species as Vorticella aquadulcis, Cyclidium glaucoma, V. convallaria, Glaucoma reniformis, and Cinetochilum margaritaceum reached their higher densities in the rainy period.

\subsection{Effect of environmental heterogeneity on protozoan communities}

We found higher abundance values of both protozoan groups related to system productivity. This was shown by larger abundance values associated to sample units with elevated nutrient concentrations and higher primary (chlorophyll) and bacterial productivity, as evidenced by the RDA.

Food availability is an important abiotic factor controlling the distribution of protozoan populations in distinct ecosystems (Pace, 1982). Velho et al. (2005) observed a positive correlation between ciliates and nutrient concentrations, suggesting that resource availability is relevant to determine the abundance distribution of these organisms. The most abundant ciliate species at the Mandacaru stream, belonging to the genera Vorticella and Cyclidium, are considered as bacterivores/algivores and bacterivores, respectively (according to Foissner and
Berger, 1996; Segovia et al., 2016). The high abundance of bacterivore ciliates seems to be widespread among streams (Segovia et al., 2016).

Moreover, many studies have demonstrated the effect of organic pollution on trophic relationships, as in ciliate protozoan communities in freshwater systems (Primc-Habdija et al., 1998; Madoni, 2005; Madoni and Braghiroli, 2007; Segovia et al., 2016). Thus, studies show a dominance of bacterivore ciliates with increasing organic load (Madoni and Braghiroli, 2007; Segovia et al., 2016).

Organic pollutants provoke an increase in phosphate and other nutrients, altering the structure of bacteria communities, inducing changes in the protists, which have bacteria as one of their main food sources (Pace, 1982). Therefore, elevated values of phosphorous and other nutrients may promote a higher availability of resources for ciliates. In this sense, ciliate and testate amoebae abundances were, in general, associated to larger values of BOD and $\mathrm{COD}$, suggesting a positive relationship between organic pollution and protozoan abundance.

\section{Conclusion}

From the results obtained in this study, we may conclude that the Mandacaru stream, although strongly influenced by anthropic action due to its location at the metropolitan region of a medium sized city, still presents an acceptable water quality, according to WQI. Furthermore, the TSI evidenced the prevalence of mesotrophic conditions in most of the sampled points and periods.

Lastly, we emphasize that protists abundance was strongly influenced by system productivity. This was evidenced by elevated protozoan densities where there was higher primary and bacterial productivity. Thus, these organisms must be considered in studies that aim at the identification of organisms that may indicate anthropic impacts and environmental quality.

\section{Acknowledgements}

We would like to thank Nupélia/PEA/PGB/UEM for logistical support. We would like to thank CAPES and CNPq for fellowships. We also thank Geziele Mucio Alves, Leilane Schwind, Diogo Castanho Amaral, Maria do Carmo Roberto, Carlos Eduardo Aguiar Soares and Lisiane Hoff for their assistance in field surveys, biological material analyzes and laboratory procedures as well as for valuable suggestions.

\section{References}

ADL, S.M., SIMPSON, A.G.B., FARMER, M.A., ANDERSEN, R.A., ANDERSON, O.R., BARTA, J.R., BOWSER, S.S., BRUGEROLLE, G., FENSOME, R.A., FREDERICQ, S., JAMES, T., KARPOV, S., KUGRENS, P., KRUG, J., LANE, C., LEWIS, L.A., LODGE, J., LYNN, D.H., MANN, D.G., MCCOURT, R.M., MENDOZA, L., MOESTRUP, O., MOZLEYSTANDRIDGE, S., NERAD, T.A., SHEARER, C.A., SMIRNOV, A., SPIEGEL, F.W. and TAYLOR, M.F.J.R., 2005. The new 
higher level classification of eukaryotes with emphasis on the taxonomy of protists. The Journal of Eukaryotic Microbiology, vol. 52, no. 5, pp. 399-451. http://dx.doi.org/10.1111/j.15507408.2005.00053.x. PMid:16248873.

AGÊNCIA NACIONAL DE ÁGUAS - ANA, 2004. Projeto de gerenciamento integrado das atividades desenvolvidas em terra na bacia do São Francisco. Sub-projeto 4.5C: plano decenal de recursos hídricos da bacia do Rio São Francisco - PbHSF (2004-2013). Brasília: SUM/ANA. Alocação de Água: Estudo Técnico de Apoio, no. 16

ALBERTI, M., 2009. Advances in urban ecology: integrating humans and ecological processes in urban ecosystems. New York: Springer.

ALMEIDA, M.A.B. and SCHWARZBOLD, A., 2003. Avaliação sazonal da qualidade das águas do Arroio da Cria Montenegro, RS com aplicação de um índice de qualidade de água (IQA). Revista Brasileira de Recursos Hidricos, vol. 8, no. 1, pp. 81-97. http:// dx.doi.org/10.21168/rbrh.v8n1.p81-97.

ALVES, G.M., VELHO, L.F.M., COSTA, D.M. and LANSACTÔHA, F.A., 2012. Size structure of testate amoebae (Arcellinida and Euglyphida) in different habitats from a lake in the upper Paraná River floodplain. European Journal of Protistology, vol. 48, no. 3, pp. 169-177. http://dx.doi.org/10.1016/j.ejop.2011.10.004. PMid:22261279.

AMERICAN PUBLIC HEALTH ASSOCIATION -APHA, 1985. Standard methods for the examination of water and waste water. Washington: American Public Health Association.

AMERICAN PUBLIC HEALTH ASSOCIATION -APHA, 2006. Standard methods for the examination of water and wastewater. New York: American Public Health Association.

ANDRIETTI, G., FREIRE, R., AMARAL, A.G.D., ALMEIDA, F.T.D., BONGIOVANI, M.C. and SCHNEIDER, R.M., 2016. Water quality index and eutrophication indices of Caiabi River, MT. Revista Ambiente \& Água, vol. 11, no. 1, pp. 162-175. http:// dx.doi.org/10.4136/1980-993X.

AUER, B. and ARNDT, H., 2001. Taxonomic composition and biomass of heterotrophic flagellates in relation to lake trophy and season. Freshwater Biology, vol. 46, no. 7, pp. 959-972. http:// dx.doi.org/10.1046/j.1365-2427.2001.00730.x.

BAGATINI, I.L., SPÍNOLA, A.L.G., PERES, B.M., MANSANO, A.S., RODRIGUES, M.A.A., BATALHA, M.A.P.L., LUCCA, J.V., GODINHO, M.J.L., TUNDISI, T.M. and SELEGHIM, M.H.R., 2013. Protozoplankton and its relationship with environmental conditions in 13 water bodies of the Mogi-Guaçu basin-SP, Brazil. Biota Neotropica, vol. 13, no. 4, pp. 152-163. http://dx.doi.org/10.1590/S1676-06032013000400016.

BEAVER, J.R. and CRISMAN, T.L., 1989. Analysis of the community structure of planktonic ciliated Protozoa relative to trophic state in Florida lakes. Hydrobiologia, vol. 174, no. 3, pp. 177-184. http://dx.doi.org/10.1007/BF00008155.

BJØRNSEN, P.K., 1986. Automatic determination of bacterioplankton biomass by image analysis. Applied and Environmental Microbiology, vol. 51, no. 6, pp. 1199-1204. PMid:16347077.

BRASIL. Conselho Nacional do Meio Ambiente - CONAMA, 2005. Resolução $n^{\circ} 357$, de 17 de março de 2005. Dispõe sobre a classificação dos corpos de água e diretrizes ambientais para o seu enquadramento, bem como estabelece as condições e padrões de lançamento de efluentes, e dá outras providências. Diário Oficial da República Federativa do Brasil, Brasilia, 18 março.
COMPANHIA DE TECNOLOGIA DE SANEAMENTO AMBIENTAL - CETESB, 2004. Relatório de qualidade das águas interiores no estado de São Paulo 2003. São Paulo: CETESB.

COMPANHIA DE TECNOLOGIA DE SANEAMENTO AMBIENTAL-CETESB, 2008. Relatório de qualidade das águas interiores no estado de São Paulo 2007. São Paulo: CETESB.

COMPANHIA DE TECNOLOGIA DE SANEAMENTO AMBIENTAL - CETESB, 2009. Relatório de qualidade das águas interiores no estado de São Paulo 2008. São Paulo: CETESB.

COMPANHIA DE TECNOLOGIA DE SANEAMENTO AMBIENTAL - CETESB, 2010. Relatório de qualidade das águas superficiais no estado de São Paulo 2009. São Paulo: CETESB.

CUNICO, A.M., FERREIRA, F.A., AGOSTINHO, A.A., BEAUMORD, A. and FERNANDES, A.C.R., 2012. The effects of local and regional environmental factors on the structure of fish assemblages in the Pirapó Basin, Southern Brazil. Landscape and Urban Planning, vol. 105, no. 3, pp. 336-344. http://dx.doi. org/10.1016/j.landurbplan.2012.01.002.

DAJOZ, R., 1973. Ecologia geral. São Paulo: Vozes.

DEBASTIANI, C., MEIRA, B.R., LANSAC-TOHA, F.M., VELHO, L.F.M. and LANSAC-TOHA, F.A., 2016. Protozoa ciliates Community structure in urban streams and their environmental use as indicators. Brazilian Journal of Biology $=$ Revista Brasileira de Biologia, vol. 76, no. 4, pp. 1043-1053. http://dx.doi.org/10.1590/1519-6984.08615. PMid:27191462.

DIAS, R.J.P., D'ÁVILA, S., WIELOCH, A.H. and D'AGOSTO, M., 2008. Protozoan ciliate epibionts on the freshwater apple snail Pomacea figulina (Spix, 1827) (Gastropoda, Ampullariidae) in an urban stream of south-east Brazil. Journal of Natural History, vol. 42, no. 19-20, pp. 1409-1420. http://dx.doi. org/10.1080/00222930802007831.

FENCHEL, T., 1987. Ecology of protozoa: the biology of freeliving phagotrophic protists. Madison: Springer-Verlag.

FOISSNER, W. and BERGER, H.A., 1996. User-friendly guide to the ciliates (Protozoa, Ciliophora) commonly used by hydrobiologists as bioindicators in rivers, lakes and waste waters, with notes on their ecology. Freshwater Biology, vol. 35, no. 1, pp. 375-482. http://dx.doi.org/10.1111/j.1365-2427.1996.tb01775.x.

FOISSNER, W., BERGER, H. and SCHAUMBURG, J., 1999. Identification and ecology of limnetic plankton ciliates. Munich: Bavarian State Office for Water Management.

FRITZSONS, E., HINDII, E.C., MANTOVANI, L.E. and RIZZI, N.E., 2003. Consequências da alteração da vazão sobre alguns parâmetros de qualidade de água fluvial. Floresta, vol. 33, no. 2, pp. 201-214. http://dx.doi.org/10.5380/rf.v33i2.2274.

FULONE, L.J., LIMA, A.F., ALVES, G.M., VELHO, L.F.M. and LANSAC-TÔHA, F.A., 2005. Composição de amebas testáceas (Protozoa-Rhizopoda) de dois córregos do Estado de São Paulo incluindo novos registros para o Brasil. Acta Scientiarum. Biological Sciences, vol. 27, no. 2, pp. 113-118. http://dx.doi. org/10.4025/actascibiolsci.v27i2.1319.

GOLTERMAN, H.L., CLYMO, R.S. and OHNSTAD, M.A.M., 1978. Methods for physical and chemical analysis of freshwater. London: Blackweel Scientific Publication.

HARFUCH, C.A.C., OLIVEIRA, F.R., MEIRA, B.R., CAGNI, G.S., SOUZA, R.F., LIZAMA, M.D.L.A.P. and VELHO, L.F.M., 2019. Qualidade da água no trecho superior da bacia do rio Pirapó: um rio urbano no sul do brasil. Revista Gestão \& Sustentabilidade 
Ambiental, vol. 8, no. 2, pp. 513-538. http://dx.doi.org/10.19177/ rgsa.v8e22019513-538.

JACKSON, D.A., 1993. Stopping rules in principal component analyses: a comparison of heuristical and statistical approaches. Ecology, vol. 74, no. 8, pp. 2204-2214. http://dx.doi.org/10.2307/1939574.

JACKSON, D.A., PERES-NETO, P.R. and OLDEN, J.D., 2001. What controls who is where in freshwater fish communities - the roles of biotic, abiotic, and spatial factors. Canadian Journal of Fisheries and Aquatic Sciences, vol. 58, no. 1, pp. 157-170. http:// dx.doi.org/10.1139/f00-239.

KISS, A.K., ACS, E., KISS, K.T. and TÖRÖK, J.K., 2009. Structure and seasonal dynamics of the protozoan community (heterotrophic flagellates, ciliates, amoeboid protozoa) in the plankton of a large river (River Danube, Hungary). European Journal of Protistology, vol. 45, no. 2, pp. 121-138. http://dx.doi. org/10.1016/j.ejop.2008.08.002. PMid:19285382.

LAMPARELLI, M.C., 2004. Grau de trofia em corpos d'água do Estado de São Paulo: avaliação dos métodos de monitoramento. São Paulo: Universidade de São Paulo. Tese de Doutorado em Ecologia Aplicada.

LAMPERT, W. and SOMMER, U., 2007. Limnoecology: the ecology of lakes and streams. New York: Oxford University Press.

LANSAC-TOHA, F.A., ALVES, G.M., VELHO, L.F.M., ROBERTSON, B.A. and JOKO, C.Y., 2008. Composition and occurrence of testate amoebae in the Curuá-Una Rervoir (State of Pará). Acta Limnologica Brasiliensia, vol. 20, no. 3, pp. 177-195.

LANSAC-TÔHA, F.A., VELHO, L.F.M., COSTA, D.M., SIMÕES, N.R. and ALVES, G.M., 2014. Structure of the testate amoebae community in different habitats in a neotropical floodplain. Brazilian Journal of Biology $=$ Revista Brasileira de Biologia, vol. 74, no. 1, pp. 181-190. http://dx.doi.org/10.1590/15196984.24912. PMid:25055100.

LANSAC-TÔHA, F.A., ZIMMERMANN-CALLEGARI, M.C., VELHO, L.F.M., ALVES, G.M. and FULONE, L.J., 2007. Species richness and geographic distribution of testate amoebae (Rhizopoda) in Brazilian freshwater environments. Acta Scientiarum. Biological Sciences, vol. 29, no. 2, pp. 185-195.

LEGENDRE, P. and LEGENDRE, L., 1998. Numerical ecology: developments in environmental modelling. Amsterdam: Elsevier.

LIPPERT, M.A.M., 2013. Ocorrência de amebas testáceas em córregos do oeste do estado do Paraná, Brasil. Maringá: Universidade Estadual de Maringá. Tese de Doutorado em Biologia Comparada.

LOBO, E. and LEIGHTON, G., 1986. Estructuras comunitarias de las fitocenosis planctonicas de los sistemas de desembocaduras de rios y esteros de la zona central de Chile. Revista de Biología Marina y Oceanografia, vol. 22, no. 1, pp. 1-29.

LOBO, E.A., CALLEGARO, V.L.M., HERMANY, G., BES, D., WETZEL, C.E. and OLIVEIRA, M.A., 2004. Use of epilithic diatoms as bioindicators, with special emphasis to the eutrophication problem of lotic systems in Southern Brazil. Acta Limnologica Brasiliensia, vol. 16, no. 1, pp. 25-40.

LOPES, M.P., MARTINS, R.T., SILVEIRA, L.S. and ALVES, R.G., 2015. The leaf breakdown of Picramnia sellowii (Picramniales: Picramniaceae) as index of anthropic disturbances in tropical streams. Brazilian Journal of Biology $=$ Revista Brasileira de Biologia, vol. 75, no. 4, pp. 846-853. http://dx.doi.org/10.1590/15196984.00414. PMid:26628230.
LYNN, D.H., 2008. The ciliated protozoa: characterization, classification, and guide to the literature. Dordrecht: Springer.

MADONI, P. and BRAGHIROLI, S., 2007. Changes in the ciliate assemblage along a fluvial system related to physical, chemical and geomorphological characteristics. European Journal of Protistology, vol. 43, no. 2, pp. 67-75. http://dx.doi.org/10.1016/j. ejop.2006.09.004. PMid:17222541.

MADONI, P., 2005. Ciliated protozoan communities and saprobic evaluation of water quality in the hilly zone of some tributaries of the Po River (northern Italy). Hydrobiologia, vol. 541, no. 1, pp. 55-69. http://dx.doi.org/10.1007/s10750-004-4667-8.

MANSANO, A.S., HISATUGO, K.F., LEITE, M.A., LUZIA, A.P. and REGALI-SELEGHIM, M.H., 2013. Seasonal variation of the protozooplanktonic community in a tropical oligotrophic environment (Ilha Solteira reservoir, Brazil). Brazilian Journal of Biology $=$ Revista Brasileira de Biologia, vol. 73, no. 2, pp. 321-330. http://dx.doi.org/10.1590/S1519-69842013000200012. PMid:23917559.

MARGALEF, R., 1963. On certain unifying principles in ecology. American Naturalist, vol. 97, no. 897, pp. 357-374. http://dx.doi. org/10.1086/282286.

MAROTTA, H., SANTOS, R.O. and ENRICH-PRAST, A., 2008. Monitoramento limnológico: um instrumento para a conservação dos recursos hídricos no planejamento e na gestão urbano-ambientais. Ambiente \& Sociedade, vol. 11, no. 1, pp. 67-79. http://dx.doi.org/10.1590/S1414-753X2008000100006.

MARTÍNEZ-FERNÁNDEZ, V., SOLANA-GUTIÉRREZ, J., GARCÍA DE JALÓN, D. and ALONSO, C., 2019. Sign, strength and shape of stream fish-based metric responses to geo-climatic and human pressure gradients. Ecological Indicators, vol. 104, no. 1, pp. 86-95. http://dx.doi.org/10.1016/j.ecolind.2019.04.076.

MCCUNE, B. and MEFFORD, M.J., 1999. PC-ORD: multivariate analysis of ecological data, version 4. Gleneden Beach: MjM Software Design.

MOERKE, A.H. and LAMBERTI, G.A., 2006. Scale-dependent influences on water quality, habitat, and fish commnities in streams of the Kalamazoo River Basin, Michigan (USA). Aquatic Sciences, vol. 68, no. 2, pp. 193-205. http://dx.doi.org/10.1007/ s00027-006-0837-2.

MUNN, M.D., BLACK, R.W. and GRUBER, S.J., 2002. Response of benthic algae to environmental gradients in an agriculturally dominated landscape. Journal of the North American Benthological Society, vol. 21, no. 2, pp. 221-237. http://dx.doi. org/10.2307/1468411

NTENGWE, F.W., 2006. Pollutant loads and water quality in streams of heavily populated and industrialised towns. Physics and Chemistry of the Earth, vol. 31, no. 15-16, pp. 832-839. http://dx.doi.org/10.1016/j.pce.2006.08.025.

OGDEN, C.G. and HEDLEY, R.H., 1980. An atlas of freshwater testate amoebae. Oxford: University Press. http://dx.doi. org/10.1097/00010694-198009000-00013.

PACE, M.L., 1982. Planktonic ciliates: their distribution, abundance, and relationships to microbial resources in a monomitic lakes. Canadian Journal of Fisheries and Aquatic Sciences, vol. 39, no. 8, pp. 1106-1116. http://dx.doi.org/10.1139/f82-148.

PATTERSON, R.T. and KUMAR, A., 2000. Assessment of arcellacean (thecamoebian) assemblages, species and strains as contaminant indicators in variably contaminated James Lake, north 
Eastern Ontario. Journal of Foraminiferal Research, vol. 30, no. 4, pp. 310-320. http://dx.doi.org/10.2113/0300310.

PAUL, M.J. and MEYER, J.L., 2001. Streams in the urban landscape. In: J.M. MARZLUFF, E. SHULENBERGER, W. ENDLICHER, M. ALBERTI, G. BRADLEY, C. RYAN, U. SIMON and C. ZUMBRUNNEM, eds. Urban Ecology: an international perspective on the interaction between humans and nature. 32th ed. New York: Springer, pp. 207-231.

PAULA, P.F. and FERREIRA, M.E.M.C., 2005. levantamento fitogeográfico preliminar no Parque do Cinquentenário em MaringáPR. Geografia: Revista do Departamento de Geociências., vol. 14, no. 1, pp. 73-86. http://dx.doi.org/10.5433/2447-1747.2005v14n1p73.

PESCE, S.F. and WUNDERLIN, D.A., 2000. Use of water quality indices to verify the impact of Cordoba city (Argentina) on Suqua river. Water Research, vol. 34, no. 11, pp. 2915-2926. http://dx.doi.org/10.1016/S0043-1354(00)00036-1.

PRIMC-HABDIJA, B., HABDIJA, I. and RADANOVIĆ, I., 1998. Seasonal changes in trophic structure of periphytic ciliates in relation to discharge regime. Internationale Vereinigung für Theoretische und Angewandte Limnologie, vol. 26, no. 3, pp. 1116-1119. http://dx.doi.org/10.1080/03680770.1995.11900893.

R CORE TEAM, 2013. $R$ : A language and environment for statistical computing. Viena: R Foudation for Statistical Computing.

REBOUÇAS, A.C., BRAGA, B. and TUNDISI, J.G., 2002. Águas doces no Brasil: capital ecológico, uso e conservação. São Paulo: Escrituras.

REYNOLDS, C.S. and DESCY, J.P., 1996. The production, biomass and structure of phytoplankton in large rivers. Archiv für Hydrobiologie, vol. 113, no. 1, pp. 161-187. http://dx.doi. org/10.1127/lr/10/1996/161.

ROSSI, A., BOSCARO, V., CARDUCCI, D., SERRA, V., MODEO, L., VERNI, F., FOKIN, S.I. and PETRONI, G., 2016. Ciliate communities and hidden biodiversity in 449 freshwater biotopes of the Pistoia province (Toscana, Italy). European Journal of Protistology, vol. 53, no. 1, pp. 11-19. http://dx.doi. org/10.1016/j.ejop.2015.12.005. PMid:26773904.

SEGOVIA, B.T., LANSAC-TOHA, F.M., DE MEIRA, B.R., CABRAL, A.F., LANSAC-TÔHA, F.A. and VELHO, L.F.M., 2016. Anthropogenic disturbances influencing ciliate functional feeding groups in impacted streams. Environmental Science and Pollution Research International, vol. 23, no. 19, pp. 2003-2016. http://dx.doi.org/10.1007/s11356-016-7185-0. PMid:27439753.

SHERR, E.B. and SHERR, B.F., 1993. Preservation and storage of samples for enumeration of heterotrophic protists. In: P. KEMP, B. SHERR, E. SHERR and J. COLE, eds. Current methods in aquatic microbial ecology. 1st ed. New York: Lewis Publishers, pp. 207-212.

SHOCHAT, E., WARREN, P., FAETH, S., MCINTYRE, N. and HOPE, D., 2006. From patterns to emerging processes in mechanistic urban ecology. Trends in Ecology \& Evolution, vol. 21, no. 4, pp. 186-191. http://dx.doi.org/10.1016/j.tree.2005.11.019. PMid:16701084.

SPERLING, M., 2007. Princípios do tratamento biológico de águas residuárias: estudos e modelagem da qualidade da água de rios. Belo Horizonte: Departamento de Engenharia Sanitária e Ambiental, Universidade Federal de Minas Gerais, 211 p.

STATSOFT, 2005. Statistica for Windows (data analysis software system), version 7.1. Tulsa: Statsoft Inc.

VELHO, L.F.M. and LANSAC-TÔHA, F.A., 1996. Testate amoebae (Rhizopodea-Sarcodina) from zooplankton of the high Paraná river floodplain, state of Mato Grosso do Sul, Brazil: II. Family Difflugidae. Studies on Neotropical Fauna and Environment, vol. 31, no. 3-4, pp. 174-192. http://dx.doi. org/10.1076/snfe.31.3.179.13342.

VELHO, L.F.M., LANSAC-TÔHA, F.M., BUOSI, P.R.B., MEIRA, B.R., CABRAL, A.F. and LANSAC-TÔHA, F.A., 2013. Structure of planktonic ciliates community (Protist, Ciliophora) from an urban lake of southern Brazil. Acta Scientiarum. Biological Sciences, vol. 35, no. 4, pp. 531-539. http://dx.doi.org/10.4025/ actascibiolsci.v35i4.18579.

VELHO, L.F.M., LANSAC-TÔHA, F.A. and BINI, L.M., 2003. Influence of environmental heterogeneity on the structure of testate amoebae (Protozoa, Rhizopoda) assemblages in the plankton of the upper Paraná River floodplain, Brazil. International Review of Hydrobiology, vol. 88, no. 2, pp. 154-166. http://dx.doi. org/10.1002/iroh.200390011.

VELHO, L.F.M., LANSAC-TÔHA, F.A. and SERAFIMJUNIOR, M., 1996. Testate amoebae (Rhizopodea-Sarcodina) from zooplankton of the Upper Paraná river floodplain, state of Mato Grosso do Sul, Brazil. I. Families Arcellidae and Centropyxidae. Studies on Neotropical Fauna and Environment, vol. 31, no. 1, pp. 35-50. http://dx.doi.org/10.1076/snfe.31.1.35.13315.

VELHO, L.F.M., PEREIRA, D.G., PAGIORO, T.A., SANTOS, V.D., PERENHA, M.C.Z. and LANSAC-TÔHA, F.A., 2005. Abundance, biomass and size structure of planktonic ciliates in reservoirs with distinct trophic states. Acta Limnologica Brasiliensia, vol. 17, no. 4, pp. 361-371.

WETZEL, R.G., 2001. Limnology. San Diego: Academic Press.

XU, K., CHOI, J.K., YANG, E.J., LEE, K.C. and LEI, Y., 2002. Biomonitoring of coastal pollution status using protozoan communities with a modified PFU method. Marine Pollution Bulletin, vol. 44, no. 9, pp. 877-886. http://dx.doi.org/10.1016/ S0025-326X(02)00090-5. PMid:12405212.

ZANINI, H.L.H.T., AMARAL, L.A., ZANINI, J.R. and TAVARES, L.H.S., 2010. Caracterização da água da microbacia do córrego Rico avaliada pelo índice de qualidade de água e de estado trófico. Engenharia Agrícola, vol. 30, no. 1, pp. 732-741. http://dx.doi.org/10.1590/S0100-69162010000400017. 